\title{
The intradiscal failure pressure on porcine lumbar intervertebral discs: an experimental approach
}

\author{
A. R. G. Araújo, N. Peixinho, A. Pinho, and J. C. P. Claro \\ Department of Mechanical Engineering, University of Minho, Guimarães, Portugal \\ Correspondence to: A. R. G. Araújo (angeloaraujo@dem.uminho.pt)
}

Received: 29 July 2015 - Revised: 10 November 2015 - Accepted: 17 November 2015 - Published: 10 December 2015

\begin{abstract}
The intervertebral disc is submitted to complex loading during its normal daily activities which are responsible for variations of the hydrostatic pressure in its structure. Thus, the determination of the magnitude of failure hydrostatic pressure is essential as a potential for the evaluation of the mechanisms that promote the weakening and the disruption of the annular fibers, commonly linked to herniation process on the spine column. However, few studies include the determination of the failure pressure on discs and the results are widely contradictory. Therefore, the objective of the present work is to determine the values of IDP that promotes the disc disruption. To achieve this goal, the tests were performed using a hydraulic cylinder that inflates the intervertebral disc. The results revealed a mean pressure failure of $0.62 \pm 0.08 \mathrm{MPa}$ for lumbar porcine samples $(n=6)$. From this approach it can be concluded that (1) the potential for disc injury may exist at low pressures for lumbar porcine discs when compared several animal and human ones; (2) the rupture of human cervical and porcine lumbar annular fibers could occur for values of intradiscal pressure that are within the physiological range.
\end{abstract}

\section{Introduction}

The intervertebral disc (IVD) is a complex and inhomogeneous structure structure composed by an inner gel-like core - the Nucleus Pulposus (NP) surrounded by a layered structure, the Annulus Fibrosus (AF). These structures are limited at the top and bottom by the Cartilaginous Endplate (CEP). The IVD allows successful load-bearing movements due to a synergetic effect of all components. The loads applied on the disc during its normal daily activities are responsible for variations of the internal disc pressure in the NP (Schechtman et al., 2006).

The internal disc pressure or intradiscal pressure (IDP) can be defined as the hydrostatic pressure presented by the NP of an healthy IVD (Claus et al., 2008). The IDP plays a key role on the IVD's ability to withstand the physiological loads (Steffen et al., 1998), being an important parameter to understand the spinal on the disc degeneration. In fact, the IDP data has been essential for prevent the spinal complaints by forming a basis for clinical advice to promote the correct sitting postures. The measurements of IDP also help to clarify the effect of the external loads on the IVD behavior (Claus et al., 2008) and to recognize the mechanism of IDP drop in disc degeneration. In addition, these data form the basis for physiotherapy and rehabilitation programs (Wilke et al., 1999).

From a biomechanical point of view, the IDP is influenced by the axial loads acting on spine (Sato et al., 1999). An increase in the compressive load applied to healthy discs is "converted" into IDP (Schechtman et al., 2006). As the NP can be considered incompressible (Castro et al., 2014), the AF bulges outwardly due to the stretch of annular fibres (Van der Veen et al., 2008) which, together with osmotic phenomenon, promotes a loss in both IVD height and volume.

The importance of IDP is reinforced due to difficult on the assessment of the disc strengthen properties (Schechtman et al., 2006). Although a simple axial compressive overload could not induce damage in an healthy disc, some movements such as compression combined with hyperflexion might generate an IDP beyond what the disc could withstand, promoting several injuries (Nachemson and Elfström, 1970; Schechtman et al., 2006). Previous studies had demonstrated that, before occurring any disc disruption, the com- 
pressive overloading provokes the vertebral endplate damage and collapse (Dolan et al., 2013; Schechtman et al., 2006). Thus, the study of the IDP is a subject of deep interest in order to determine its contribution for IVD injury.

The determination of the magnitude of failure IDP is also essential as a potential parameter for the evaluation of the mechanisms that promote the weakening and the disruption of the annular fibers (Iencean, 2000). Once combined with the traditional provocative discography, the IDP monitoring represents an important way to determine the clinical significance internal disc disruption (Menkowitz et al., 2005).

Few studies include the determination of the failure pressure of the IVDs (Iencean, 2000; Menkowitz et al., 2005; Schechtman et al., 2006; Veres et al., 2010). Schechtman et al. (2006) investigated the intrinsic failure strength of the intact bovine caudal disc using an hydraulic inflation actuator. A colored hydrogel was injected into NP under monitored pressure. It was found a mean hydrostatic failure pressure of $18 \pm 3 \mathrm{MPa}$. This method allowed understanding the alterations of the intrinsic disc strength associated with prior loading history or degeneration. However, it does not give information about the microstructural behavior of inner annular fibers after the inflation. Later, Veres et al. (2010) used the same technique performed by Schechtman et al. (2006) to investigate the role of high IDP in the disruption of the annular fibers of the ovine lumbar IVDs. This team included the analysis of the AF damage after pressure insertion by a microstructural investigation. It was found a mean failure pressure of $14.1 \pm 3.9$. It was also reported that posterior annular region is more susceptible to disruption than the other disc regions, due to its inability to distribute hydrostatic pressures circumferentially.

However, other studies showed that the IVD's injuries could be induced at lower IDPs. Iencean (2000) developed an experimental device for determine the rupture IDP of lumbar intervertebral discs, consisting of a source of pressure, connected to a tube introduced into the IVD through a tunnel drilled in the body of the subjacent vertebra by CEP access. The results revealed that the rupture was reached for IDP ranged from 0.75 to $1.3 \mathrm{MPa}$ for neutral posture and a maximum rupture IDP in anterior flexion of 1.2 MPa. Later, using a $25 \mathrm{G}$ needle for the insertion of pressure on cervical discs, Menkowitz et al. (2005), reported a mean intradiscal rupture pressure of $0.28 \mathrm{MPa}$ (range 0.1-1.18 MPa). Both studies have demonstrated that the rupture of human cervical and porcine lumbar annular fibers could occur for IDPs within the physiological range.

Therefore, the objective of the present work is to bring an additional insight about the magnitude of IDP that leads to AF disruption. To achieve this goal, the tests were performed using a hydraulic cylinder that inflates the IVD with glycerin, while a porcine lumbar disc is compressed. The pressure was monitored by a digital manometer and the maximum point of pressure was considered as the rupture point. This inflation method, combined with a pre-defined compression, allows not only inducing a pure hydrostatic loading due to glycerin insertion, but also producing a hydrostatic component and an environment in which the disruption of the disc in-vivo could occur.

\section{The materials and methods}

\subsection{Motion segment collection and preservation}

After being collected, the lumbar spines from pigs with 18 months old were immediately sectioned into motion segments (MS) visible in the Fig. 1 (Araújo et al., 2014; Campbell-Kyureghyan et al., 2011). Posteriorly, the MS were sealed in plastic bags and frozen at $-20^{\circ} \mathrm{C}$, until the day prior to mechanical testing, minimizing the tissue dehydration. This procedure was adopted since dead and frozen storage presents a negligible effect in mechanical properties of the spine (Adams et al., 1996). Before start any mechanical test, samples were hydrated with $12 \mathrm{~h}$ with phosphate buffer saline solution in order to prevent segment desiccation.

\subsection{Pressurization configuration used to determine the failure intradiscal pressure}

The schematic representation of the apparatus for the inducement of internal disc pressure is presented in Fig. 2.

The pressure is inserted in the inner disc region using a hydraulic cylinder. The pressure generating apparatus consists of a hydraulic cylinder, with a coupled lever that allows controlling the pressure exerted on the system. The injected pressure is assessed by a digital manometer incorporated in the system - the electronic pressure sensor PP7553, from IFM ${ }^{\circledR}$. This manometer is connected with the LineRecorder ${ }^{\circledR}$ software that allowed registering the pressure acting in the IVD as function of time. The principle of function of each failure pressure test is simple. First, all system was filled with glycerin, which is inserted in the system by the pressure exerted by the hydraulic cylinder. The glycerin was selected as testing fluid since it presents relative higher density and viscosity than water $\left(1.261 \mathrm{~g} \mathrm{~cm}^{-3}\right.$ and $1499 \mathrm{cP}$ at $20^{\circ} \mathrm{C}$, respectively). To ensure that the entire system was filled with the liquid, and so, the value of failure pressure was not affected by air bubbles in the tubes, a "bleeding" tap was included in the stainless steel basis (Fig. 2). The procedure for air bubbles removal was simple: after the placement of the motion segment on the system, the tap is opened and the glycerin is forced to enter into the system by the suction effect promoted by the lever movement. Then, the fluid was poured by the tap until ensuring that the system presents no air bubbles. Finally, the tap is closed and the system is ready to be submitted to test. 


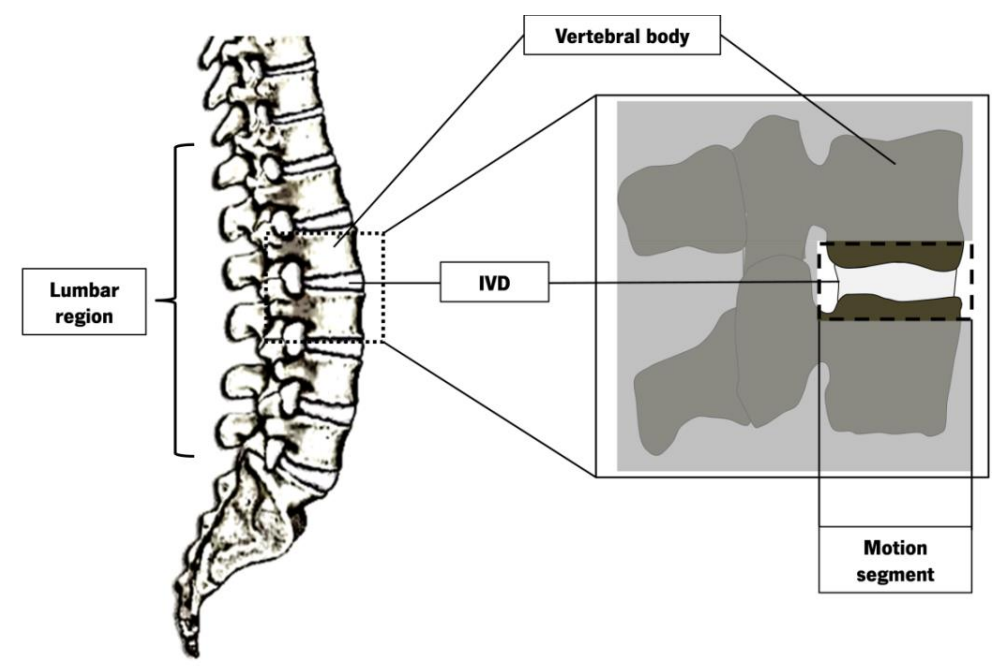

Figure 1. Schematic representation of the motion segment preparation. This structure is highlighted by the dashed rectangle. To obtain a motion segment, two vertebrae are cut parallel and transversely, obtaining an assemble composed by two half vertebra with a disc in between.

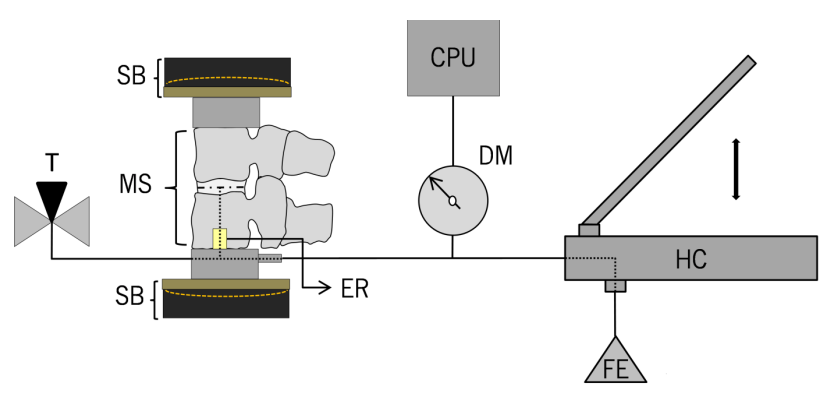

Figure 2. Failure pressure tester. The apparatus is composed by: SB - Spherical Axial Bearing; ER - Epoxy resin; MS - Motion Segment; CPU - Personal Computer; DM - Digital Manometer; T - Tap; HC - Hydraulic cylinder; T - "Bleeding" Tap; FE - Fluid Entrance.

\subsection{Motion segment attachment}

The vertebral bone is a highly porous structure, in which the fluids easily outflow from its inner region to the outer one (Bronner et al., 2010), being extremely hard to tight a screw in it. To effectively overcome this obstacle, a $9 \mathrm{~mm}$ diameter and $2 \mathrm{~mm}$ height hole as drilled in the top vertebra hole of the MS, in order to fill it with an epoxy resin with fast curing. Consequently, a $4 \mathrm{~mm}$ pilot hole was carefully drilled longitudinally through the resin and vertebrae until a sudden change in the structure resistance. This change of resistance indicates the point of contact between CEP and the NP.

The MS was then attached to pressure apparatus, in case to a homemade cylindrical stainless steel bottom plate, by a self-tapping steel screw (Fig. 3). The length of the selftapping screw was $20 \mathrm{~mm}$, presenting two threads: a section of $10 \mathrm{~mm}$ height and $7 \mathrm{~mm}$ diameter drywall screw thread to a drill in the vertebra and a region of $10 \mathrm{~mm}$ height and $5 \mathrm{~mm}$ (a)

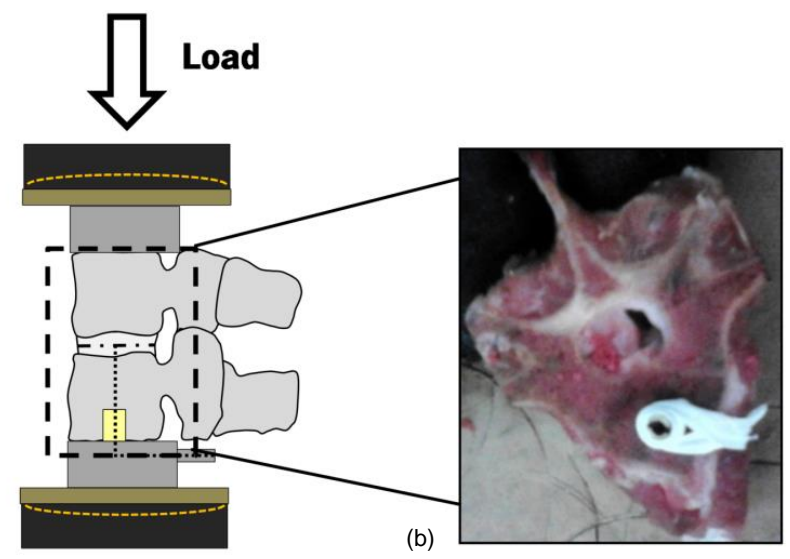

Figure 3. Motion segment attachment. (a) The MS was placed between two plates of Instron ${ }^{\circledR} 8874$, being subjected to compression. The bottom plate is drilled, allowing the fluid passage from pressure apparatus to the hollowed screw. The attachment is done on epoxy resin (yellow region), to provide a better adhesion of the screw; (b) top vision of a drilled MS, with the presence of the selftapping screw on the vertebral body.

diameter to attach to the stainless steel plate. This screw also presents a drilled hollow along its entire length, with an internal bore of $1.5 \mathrm{~mm}$ diameter that allows the fluid passage. Then, the screw was tightened until reaching the contact point between CEP and the NP. An O-Ring was placed on a cylindrical stainless steel bottom plate (around the selftapping screw), in order to prevent fluid leakage. The compressive loading was exerted in the top of the other vertebral body. The MS samples were carefully aligned according a pre-defined system of axis, using spherical axial bearing system. This alignment ensures that all discs are placing in the 


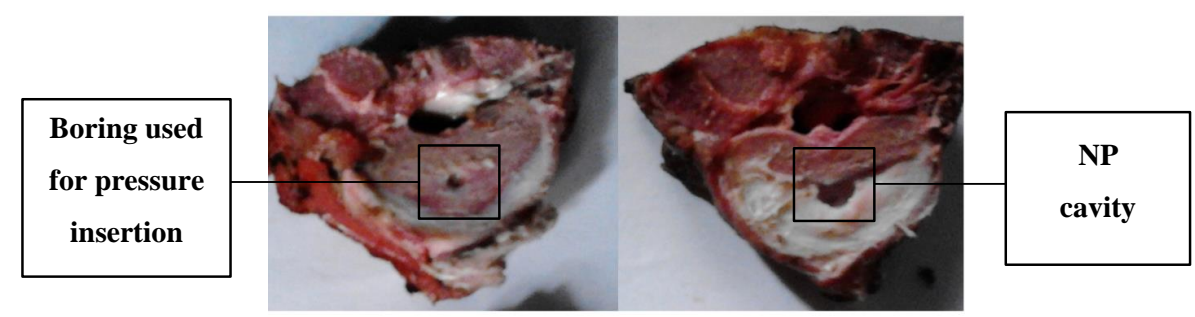

Figure 4. Images of MS sawed transversally after testing. It is visible the presence of a cavity in the NP region, indicating the pressurizing zone.

same position and compressed along the same plane, avoiding errors associated to misplacement of the samples.

The preliminary trials have failed on containing the insertion of glycerin in the nuclear cavity. In fact, during the initial tests, using only fast curing resin as interface between the screw and the MS, the fluid flowed out from the drilled vertebrae motion segment, by the screw insertion. Six motion segments were used during preliminary trials for testing the failure IDP. Then, the approach was improved by using a thin film of silicone in the interface vertebrae-stainless steel base.

\subsection{Experimental procedure}

A compressive axial displacement of $1 \mathrm{~mm}$ was imposed in the top of MS in order to either avoid the longitudinal expansion of the IVD during the pressurization test and to simulate the in-vivo confined conditions (Schechtman et al., 2006). After impose this displacement, the MS is pressurized by the descendent movement of the lever. These samples were pressurized during a period of approximately $10 \mathrm{~s}$, which represents the maximum time that all samples needed to reach failure after the application of an external pressure source. During this period, the Line Recorder ${ }^{\circledR}$ software allowed to monitored and save the values of IDP as function of time. Ten motion segments were used for the monitoring of the failure IDP.

This study had neglected the effect of the pressure drag on the walls during the fluid passage in both tubes and screw. Thus, it is assumed that the pressure read on the pressure sensor corresponds to the real inflation pressure of the IVD.

After all tests, each MS was sectioned transversally in the IVD region, in order to assess if it was pressurized in the nuclear region. The segmented disc was photographed and the final area of each IVD determined using the image processor Image Pro Plus $4.6^{\circledR}$.

\section{Results}

\subsection{Visual inspection of the pressurized motion segment}

The images were taken to assess the functionality of pressurizing system and, in addition, to validate the results. Six specimens were pressurized in the NP region, which is visi-

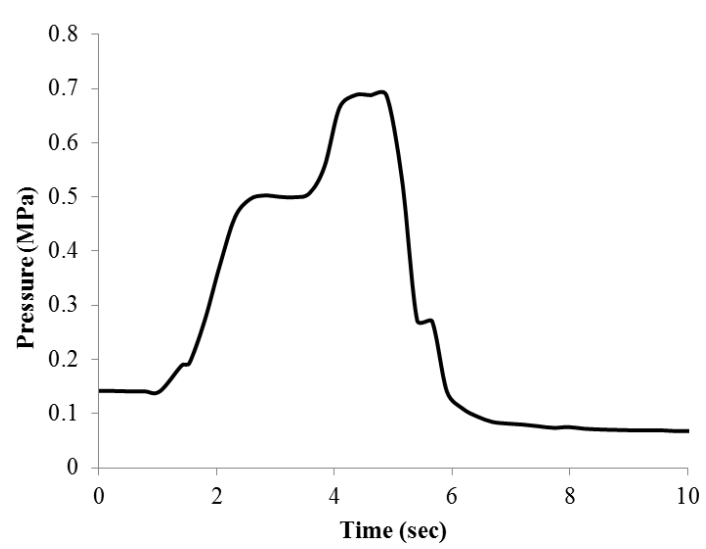

Figure 5. The characteristic response of MS to a failure IDP event (in $\mathrm{MPa}$ ) as function of time (in s).

ble in Fig. 4. Thus, six motion segments were considered for further analysis.

In this study only tests revealing a complete containing of the IVD inflation, where no leakage or liquid tear in the vertebral body, were considered. Three specimens were discarded as it was not detected a signal of external NP pressurization. Leakage in the tubes was also found in one sample.

The average disc area and height were $917.2 \pm 107.0 \mathrm{~mm}^{2}$ and $5.97 \pm 0.63 \mathrm{~mm}$, respectively.

\subsection{Failure pressure in the motion segment}

The criteria of failure in the MS structure, due to the insertion of IDP using an external source, comprises two evident phenomena: (1) the flood of the glycerin from any motion segment region, with exception for the top region of both vertebral bodies, and (2) a significant drop in the pressure detected by the digital manometer. A representative example of the curve of a failure IDP curve as function of time is depicted in Fig. 5.

The values of failure IDP together with the description of the failure in the specimen after visual inspection of the six motion segments that matched the criteria for failure are described in Table 1. In all the cases a substantial drop of pres- 
Table 1. Failure pressure (maximum in the curve IDP vs. time) for the 6 motion segments that presents external indicators of rupture, such as sudden pressure drop, leakage in the vertebra region or in spinal cord (fluid outflowing from the spinal cord channel) or tears formed on the annular region or also on the top and bottom parts of annular region (Cartilaginous Endplate region).

\begin{tabular}{lcl}
\hline $\begin{array}{l}\text { Motion } \\
\text { segment }\end{array}$ & $\begin{array}{c}\text { Failure } \\
\text { pressure } \\
\text { (MPa) }\end{array}$ & Description of the failure on the specimen after visual inspection \\
\hline 1 & 0.58 & - Moderate leakage around the screw region in the vertebra \\
- A little tear formed in the annular region
\end{tabular}

sure was visible after reach the maximum value of IDP that corresponds to the failure pressure.

Several events can be identified during the process of MS failure due to the insertion of an external pressure source. In all discs, an immediate glycerine leakage was visible after reaching the maximum value of pressure. Moreover, there was not possible to identify a pattern between the IDP failure and the place of glycerine outflow, being visible a wide range of rupture regions in the motion segment instead.

On Fig. 6 diverse examples of MS failure are exposed. The images document the different type of rupture occurring in each MS submitted to failure IDP values.

\section{Discussion}

Despite the panoply of mechanical tests intending to elucidate the contribution of loads to MS collapse, the magnitude of IDP that led to the MS rupture remains still unclear. The measurement of the internal pressure that leads to disc rupture is important not only to understand the mechanisms of IVD failure but also for the design of new implants for NP replacement. In fact, the fiber orientation of the AF is able to withstand the hoop stresses generated hydrostatic pressure in the healthy conditions (Inoue and Espinoza Orías, 2011). When the NP is removed, the outer region of the AF continues to bulge outward during the application of axial loading; conversely, the inner region bulges toward the center of the IVD (Goins et al., 2005; Meakin and Hukins, 2000). Thus, these implants should be able to exert a prescribed pressure on the inner AF walls and this pressure should be able to keep the biomechanical characteristics of the remaining disc, avoiding the disc degeneration.
Based on the method developed by Schechtman et al. (2006) to measure the failure strength on bovine caudal disc, this work determined the failure strength of the porcine MSs under an imposed IDP, using the a cartilaginous endplate access.

The definition of failure of the present work contemplates the flood of the glycerin from any region motion segment together with a significant drop in the pressure detected by the digital manometer. This consideration arises from the fact that, when a failure pressure is imposed in a MS, a chaotic effect is detectable in the whole MS structure. No localized region was detected or identified as a typical region of disc rupture after the insertion of an external pressure, indicating that there was a redistribution of the IDP in the inner region of the multilayered AF, i.e. in the contact zone between NP and AF. Thus, the collapse of the IVD structure is not a local but a generalized event.

Concerning to quantitative results, this study reports a mean pressure failure of $0.62 \pm 0.08 \mathrm{MPa}$ for lumbar porcine samples. The comparison between these results with the failure pressure of the annular fibers reported in previous studies for several MS models (Table 2) reveals that the values documented on this report are appreciably lower than the more recent studies, performed by Schechtman et al. (2006) and Veres et al. (2010). In fact, several studies reported the magnitude of the rupture values obtained in this study as within the normal range of physiological IDP for human samples (Claus et al., 2008; Dennison et al., 2008; Sato et al., 1999; Wilke et al., 1999).

Schechtman et al. (2006) investigated the intrinsic failure strength of the intact bovine caudal disc under inflation, injecting a colored gel with a hydraulic actuator: the mean hy- 


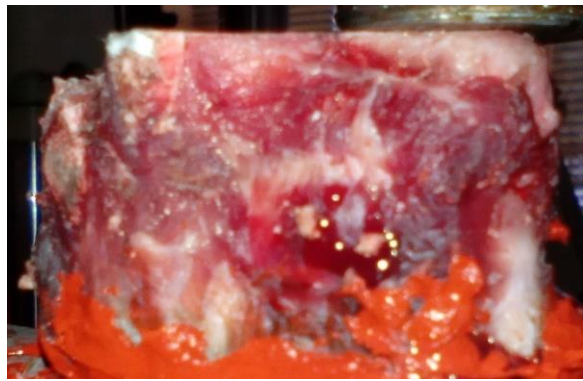

(a) Leakage of fluids from the annular region. Two motion segments failed in this manner.

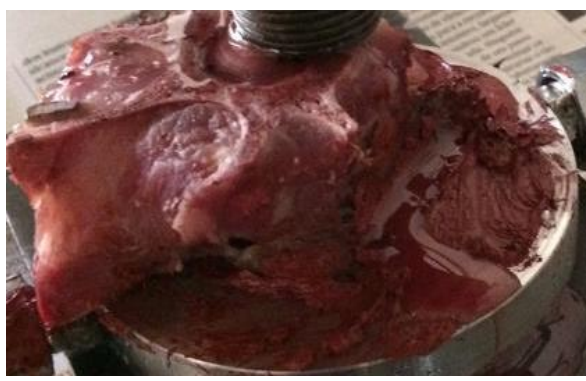

(c) Moderate leakage around the screw region in the vertebrae (bottom vertebrae). Two motion segments failed in this manner.

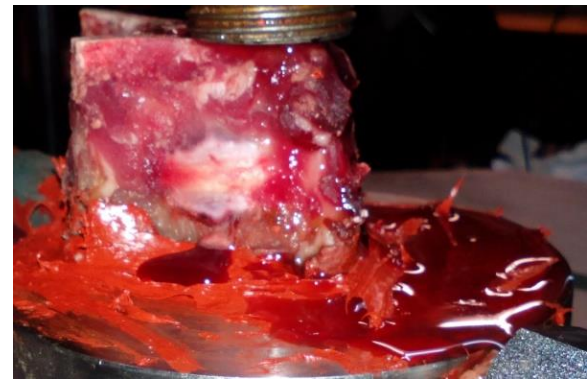

(a) Severe leakage around the screw region in the vertebrae (bottom vertebrae) and in the annular region. Slight leakage around the spinal cord (fluid outflowing from the spinal cord channel). One motion segment failed in this manner.

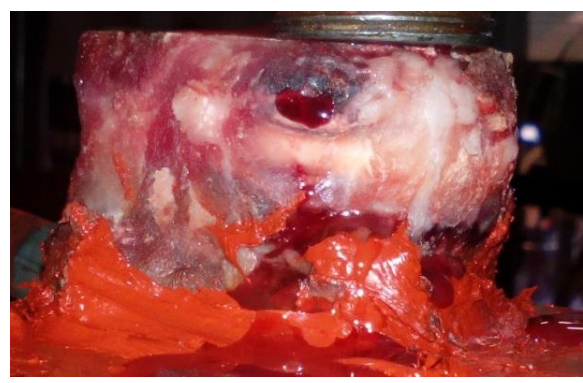

(d) Little tears formed in the top and bottom parts of annular region (CEP region). One motion segment failed in this manner.

Figure 6. Representative images of different configurations of failure in MSs, during the inflation procedure.

Table 2. Recent data about failure pressure data for different IVD models.

\begin{tabular}{llll}
\hline Author & Year & Models & $\begin{array}{l}\text { Mean failure pressure } \\
(\mathrm{MPa})\end{array}$ \\
\hline Iencean & 2000 & Lumbar human & 0.75 to 1.3 \\
Menkowitz & 2005 & Cervical human & 0.28 (min-max: 0.1-1.18) \\
Schetchman & 2006 & Caudal bovine & $18 \pm 3$ \\
Veres & 2010 & Lumbar ovine & $14.1 \pm 3.9$ \\
\hline This study & 2015 & Lumbar porcine & $0.62 \pm 0.08$ \\
\hline
\end{tabular}

drostatic failure pressure was found to be $18 \pm 3 \mathrm{MPa}$. Later, Veres et al. (2010), using the same technique performed by Schechtman et al. (2006) to investigate the role of high IDP on annular fibers disruption in ovine lumbar IVDs reported a the mean failure pressure of $14.1 \pm 3.9$. According with the latest developments about the failure pressure of disc, it would be expectable a higher value for the rupture of porcine discs, as the magnitudes presented by these authors are decidedly superior from those documented in this work.

The present results are within the same magnitude of values documented in two works performed with human lumbar (Iencean, 2000) and cervical IVDs (Menkowitz et al., 2005).
Iencean (2000), reported a rupture pressure up to $1.3 \mathrm{MPa}$ after inflating the IVD with compress air through a tunnel drilled in the body of the subjacent vertebra via CEP access; Menkowitz et al. (2005) claimed a mean intradiscal rupture pressure of $0.28 \mathrm{MPa}$ (range $0.1-1.18 \mathrm{MPa}$ ), using a $25 \mathrm{G}$ needle for the insertion of a contrast dye, with IDP monitoring during time. These studies indicate two important facts: (1) the potential for iatrogenic disc injury may exist at low pressures for lumbar porcine IVDs when compared with samples presented in Table 2; (2) the rupture of human cervical and porcine lumbar annular fibers could occur for IDPs within the physiological range, showing that the injury on these structures could be induced at significantly lower pressures.

The disparities in terms of failure pressure, presented in Table 2, could be related to several phenomena. This study had limited the expansion of the MS in terms of height, imposing a permanent $1 \mathrm{~mm}$ displacement to each disc sample while the pressurization test occured. The particular case of the use of caudal discs as model for the human lumbar disc is an option that has been questioned at several levels, as they presents different mechanical loading relative to human lumbar spine together with different composition and metabolism and different anatomy (Alini et al., 2008). The 
caudal bovine samples presents a nearly cylindrical shape (Schechtman et al., 2006), neglecting the possible existence of critical point of the hydrostatic pressures distribution in the IVD. This misassumption could lead to divergent values concerning to which is expected for human discs. Thus, the present work was conducted with lumbar porcine samples since they were considered was geometrically and morfologically more similar to the human ones, and so, a more suitable choice for mechanical studies on the IVD (Alini et al., 2008). Moreover, the different techniques of measure the failure pressure may compromise the reliability of the results. Several approaches were analyse and their weaknesses should not be neglected when a comparison is made.

This work itself present some limitations as the cadaveric study was limited by sample size or the criteria defined for MS failure. In fact, in this study the real values of rupture could be hidden by the flood of glycerin from the screwed vertebra. However, the samples where the rupture had only ocurred in the screwed vertebra were discarded and, for the quantitative analysis, only the samples with an additional point of rupture were considered. Moreover, it does not contemplate a microstructural analysis of the failure mechanisms. The rupture ocurring in the cited articles neglected the shape differences in samples, in what concerns to the critical points of failure, that are different between specimens. For a complete understanding of the process of IVD failure in compression, the phenomena ocurring on annular region and on the annular wall-endplate must be assessed, as the latter represent a typical critical point for disc rupture in compression (Adams, 2002; Schechtman et al., 2006). In addition, the data provided on this report is a result of an inflation after pre-defined compression of the IVD. Another types of efforts and loadings, such as flexion or rotation must be, together with disc inflation, must be considered for the rupture analysis.

\section{Conclusions}

In this study, the values of IDP that leads to disc rupture were determined by inflating porcine IVDs with glycerin. The experimental findings provide new insights about the mechanisms of disc failure, bringing an important addition for the validation of the constitutive models as well as to stimulate the development of more reliable solutions to replace the IVD. The main finding of this approach is that the failure could occur for a magnitude of IDP that could be found during daily activities. In light of this date, it can be concluded that the critical IDP pressure is an very specific characteristic, probably depending on compositional factors and a range of features related with specimens particularities. Moreover, this set of new experimental data should be considered on the design of more efficient solutions for the nucleus pulposus replacement, as the maintenance of a prescribed hydrostatic pressure in the inner walls of the AF is essential for keep the physiological conditions of the IVD.

However, care must be taken on the extrapolation of these results to the failure IDP in human samples. Upcoming studies should include numerical or optimization methods, in order to monitoring the factors that could induce divergences in the results, helping to clarify the magnitude of the annular failure strength in the human discs. 
Appendix A: Acronyms list

Table A1. List of acronyms.

\begin{tabular}{ll}
\hline AF & Annulus Fibrosus \\
CEP & Cartilaginous Endplate \\
IVD & Intervertebral Disc \\
IDP & Intradiscal Pressure \\
MS & Motion Segment \\
NP & Nucleus Pulposus \\
\hline
\end{tabular}


Acknowledgements. This work was funded by the project "NP Mimetic - Biomimetic Nano-Fibre Based Nucleus Pulposus Regeneration for the Treatment of Degenerative Disc Disease", financed by the European Commission under FP7 (grant NMP-2009SMALL-3-CP-FP 246351).

The authors also express their gratitude to Indústria de Carnes do Minho (ICM) - Primor Group - for the possibility of collecting spine column samples in their facilities.

Edited by: P. Flores

Reviewed by: P. Flores, A. Castro, and another anonymous referee

\section{References}

Adams, M. A.: The Biomechanics of Back Pain, Churchill, Livingstone, 2002

Adams, M. A., McNally, D. S., and Dolan, P.: "Stress" distributions inside intervertebral discs. The effects of age and degeneration, J. Bone Joint. Surg. Br., 78, 965-72, 1996.

Alini, M., Eisenstein, S. M., Ito, K., Little, C., Kettler, A. A., Masuda, K., Melrose, J., Ralphs, J., Stokes, I., and Wilke, H. J.: Are animal models useful for studying human disc disorders/degeneration?, Eur. Spine J., 17, 2-19, 2008.

Araújo, A., Peixinho, N., Pinho, A., and Claro, J. C. P.: A Novel Methodology to Assess the Relaxation Rate of the Intervertebral Disc by Increments on Intradiscal Pressure, Appl. Mech. Mater., 664, 379-383, 2014.

Bronner, F., Farach-Carson, M. C., and Roach, H. I.: Bone and Development, Springer Science \& Business Media, SpringerVerlag, London, 2010.

Campbell-Kyureghyan, N. H., Yalla, S. V., Voor, M., and Burnett, D.: Effect of orientation on measured failure strengths of thoracic and lumbar spine segments, J. Mech. Behav. Biomed. Mater., 4, 549-557, 2011.

Castro, A. P. G., Wilson, W., Huyghe, J. M., Ito, K., and Alves, J. L.: Intervertebral disc creep behavior assessment through an open source finite element solver, J. Biomech., 47, 297-301, 2014.

Claus, A., Hides, J., Moseley, G. L., and Hodges, P.: Sitting versus standing: does the intradiscal pressure cause disc degeneration or low back pain?, J. Electromyogr. Kinesiol., 18, 550-558, 2008.

Dennison, C. R., Wild, P. M., Byrnes, P. W. G., Saari, A., Itshayek, E., Wilson, D. C., Zhu, Q. A., Dvorak, M. F. S., Cripton, P. A., and Wilson, D. R.: Ex vivo measurement of lumbar intervertebral disc pressure using fibre-Bragg gratings, J. Biomech., 41, 221225,2008
Dolan, P., Luo, J., Pollintine, P., Landham, P. R., Stefanakis, M., and Adams, M. A.: Intervertebral disc decompression following endplate damage: implications for disc degeneration depend on spinal level and age, Spine, 38, 1473-181, 2013.

Goins, M. L., Wimberley, D. W., Yuan, P. S., Fitzhenry, L. N., and Vaccaro, A. R.: Nucleus pulposus replacement: an emerging technology, Spine J., 5, 317S-324S, 2005

Iencean, S. M.: Lumbar Intervertebral Disc Herniation Following Experimental Intradiscal Pressure Increase, Acta Neurochir., 142, 669-676, 2000.

Inoue, N. and Espinoza Orías, A. A.: Biomechanics of intervertebral disk degeneration, Orthop. Clin. North Am., 42, 487-99, 2011.

Meakin, J. R. and Hukins, D. W. L.: Effect of removing the nucleus pulposus on the deformation of the annulus fibrosus during compression of the intervertebral disc, J. Biomech., 33, 575-580, 2000.

Menkowitz, M., Stieber, J. R., Wenokor, C., Cohen, J. D., Donald, G. D., and Cresanti-Dakinis, C.: Intradiscal pressure monitoring in the cervical spine, Pain Physician, 8, 163-166, 2005.

Nachemson, A. and Elfström, G.: Intravital dynamic pressure measurements in lumbar discs. A study of common movements, maneuvers and exercises, Scand, J. Rehabil. Med. Suppl., 1, 1-40, 1970.

Sato, K., Kikuchi, S., and Yonezawa, T.: In vivo intradiscal pressure measurement in healthy individuals and in patients with ongoing back problems, Spine, 24, 2468-2474, 1999.

Schechtman, H., Robertson, P. A., and Broom, N. D.: Failure strength of the bovine caudal disc under internal hydrostatic pressure, J. Biomech., 39, 1401-1409, 2006.

Steffen, T., Baramki, H. G., Rubin, R., Antoniou, J., and Aebi, M. Lumbar intradiscal pressure measured in the anterior and posterolateral annular regions during asymmetrical loading, Clin. Biomech., 13, 495-505, 1998.

Van der Veen, A. J., Mullender, M. G., Kingma, I., van Dieen, J. H., Van, J. H., and Smit, T. H.: Contribution of vertebral [corrected] bodies, endplates, and intervertebral discs to the compression creep of spinal motion segments, J. Biomech., 41, 1260-1268, 2008.

Veres, S. P., Robertson, P. A., and Broom, N. D.: The influence of torsion on disc herniation when combined with flexion, Eur. Spine J., 19, 1468-1478, 2010.

Wilke, H. J., Neef, P., Caimi, M., Hoogland, T., and Claes, L. E.: New in vivo measurements of pressures in the intervertebral disc in daily life, Spine, 24, 755-762, 1999. 